\title{
DEVIATION OF DEFORMATION OF THE SCREEN Elements Through CirCUlation IN THE NeWSPAPER PRINTING
}

\author{
KUMPAR, D.; ZJAKIC, I. \& BATES, I.
}

Abstract: Consumers and markets that participate in the consumption of graphic products from year to year set new requirements and standards on quality of printing product. During the daily production screen elements are exposed to a number of different influences that can lead to distortions. With the appearance of deformation screen elements there are a difference in the surface screen elements between theoretical and actual size. Determining deformation of the screen element is done using the different of control strips. In this paper we will examine the deviation of the screen elements through the circulation in the newspaper press. Measurements with graphics and diagrams will show the deviation of the screen element throughout the entire circulation. Establishing tolerances of screen elements in daily circulation shall be done with densitometer "GretagMacbethSpectroEye" on control strips in the newspaper.

Key words: deformation, screen element, printing, quality
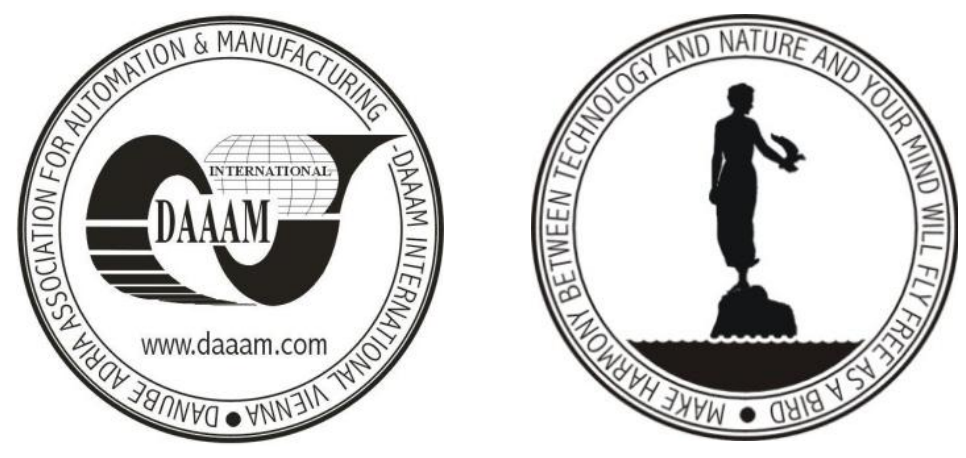

Authors' data: B.Sc. Graph. Eng. Kumpar, D[ejan]; Prof. Ass. Zjakic, I[gor]; B.Sc. Graph. Eng. Bates, I[rena], Faculty of Graphic Arts, Getaldiceva 2, 10000, Zagreb, HR,dkumpar@gmail.com, zjakic@grf.hr, irena.bertic@grf.hr

This Publication has to be referred as: Kumpar, D[ejan]; Zjakic, I[gor] \& Bates, I[rena] (2010). Deviation of Deformation of the Screen Elements Through Circulation in the Newspaper Printing, Chapter 34 in DAAAM International Scientific Book 2010, pp. 357-366, B. Katalinic (Ed.), Published by DAAAM International, ISBN 978-3-901509-74-2, ISSN 1726-9687, Vienna, Austria DOI: $10.2507 /$ daaam.scibook.2010.34 
Kumpar, D.; Zjakic, I. \& Bates, I.: Deviation of Deformation of the Screen Elemen...

\section{Introduction}

As the printing quality is one of the most important factors that define the final quality of the printed products, we can say that the deformation of the screen elements is one of the most important parameters that can affect the lower quality of graphics products.

When it comes to newspaper printing and quality within the newspaper production, one of the unavoidable facts is deformation of the screen element. One of the conditions on the quality of any offset technique is to maintain a constant quality throughout the circulation. Constant print quality can be achieved only by controlling the most important parameters that may aggravate its stability.

It is very difficult to classify all the parameters that can and do affect on print quality, but it is certain that the dot gain is surely one of the most important parameters that define the quality of printing product. One of the characteristics in the newspaper production, that may affect the quality of printing products and deviations from the value of dot gain, is the use of inadequate amounts of certain materials. Large fluctuations in the use of ink and fountain solution can lead to increasing value of dot gain elements. The consequences of increase of values of dot gain elements are reduction of the relative contrast and thus causing a decline in the quality of a given product.

\section{Theoretical part}

Dot gain is directly related to the number of parameters that can influence to deviation. However, one of the most important parameters of the printing quality are the defined and controlled dot gain values which appeared because of the deformations of the screen element during the whole printing production.

Things that can influence to deformation of dot gain:

Ink: tack and viscosity, ink/water balance, pigment strength, temperature, ink-film thickness (print density)

Paper: brightness, whiteness, opacity, porosity/holdout, smoothness, web tension

Fountain Solution: $\mathrm{pH} /$ conductivity, hardness of incoming water, type of dampening system, formulation

Blanket: compressibility, age, tension, surface characteristics

Plate: exposure time and vacuum, processing, reactions (age, light, chemical)

Rollers:hardness of material, settings, glazing

Speed: low/high

Packing: over/under

Coverage: light/heavy

The quality of screen elements defines the sharpness of detail on reproduction. Raster can be divided into Amplitude Modulated screening (AM), Frequency Modulated screening (FM) and Hybrid screening. Using the screening process is necessary if one wants to achieve the impression of several tones with an equal layer of ink on the printing substrate. 


\subsection{Dot Gain}

Dot Gain can easily be explained, like the difference between the size of the printed dot and the size of the dot that is required to be reproduced. Based on the theory of densitometry, there are a number of process attributes that can be assessed. The most important is tonal value (Dot Gain). To understand Dot Gain, we need to refer to the principle of half toning, which is fundamental to all the printing processes. Dot gain will always occur, but regardless of the process, it will need to be controlled or corrected to obtain predictable colour reproductions.

A tone reproduction curve gives to the relationship between tonal value and dot gain. Most of the information or detail, in printed halftone pictures, is carried in the tonal or tinted areas. Measuring dot gain at $25 \%, 50 \%$ and $75 \%$ for each colour is a quick indication of tone reproduction quality. Dot gain can be measured with densitometers or spectrophotometers. (Verikas \& Bacauskiene, 2008).

The deformation of the screen element can be divided into two components mechanical dot gain and the optical dot gain. Factors, which can deform the screen element during the production, influence on the screen element so that it increases (positive deformation) or decreases (negative deformation) in relation to the theoretical surface.

\subsection{Mechanical Dot Gain}

Mechanical deformation of dot gain is the physical ink spread that is mainly affected by the amount of ink emulsification, its viscosity and tack, the pressure involved during the transfer, and the substrate properties. Environmental temperature and press speed also have an effect on mechanical deformation of dot gain.

Deformation from increasing the screen element - the strain is visible when the screen elements have a fuller impression on the print sample, than on the printing form. The cause of such deformities could be the amount of colour on the screen element during printing or printing materials, chemical characteristics of inks, etc.

Deformation from reducing the screen element - it is a very rare type of deformation. An example of such a deformation would be in the case that the screen element is smaller on print sample, than on a print form.

Slurring - the change in the shape of the screen element from circular to an elongated oval shape. Slur is the deformation of a dot due to the surface speed difference of two cylinders. It is elongated in the direction of the press or opposite to print direction. The difference in speed causes the dot to elongate in the printing direction.

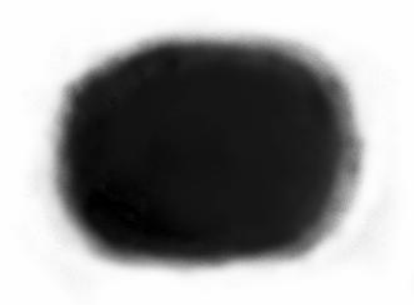

Fig. 1. Dot gain deformation with influence of slurring 
Kumpar, D.; Zjakic, I. \& Bates, I.: Deviation of Deformation of the Screen Elemen...

Doubling - is an appearance that can be seen, when next to the screen element appears a lower trace of a screen element. It is caused by the contact of cylinders with a sufficiently stretched rubber sleeve or at an irregular thickness substrate under the rubber cover. Doubling also occurs due to poor quality of paper and inadequate conditions within the printing house, which causes deformation of paper (Preston \& Gate, 2005),

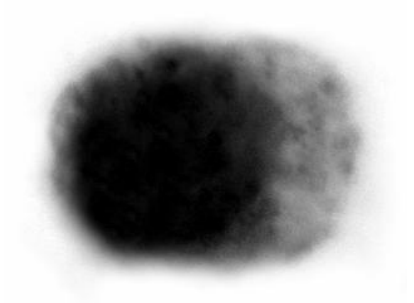

Fig. 2. Deformation of dot gain with doubling

Smudging - occurs when it comes to smearing ink from the screen element. It is formed by mechanical action after printing. It is due to a large drift of the total amount of ink on the paper when the following colour can't be received onto the paper surface, but smears on it. That happens because of insufficient absorption of the printing material. That can also occur due to improper dosing of fountain solution. In very small quantities of fountain solution plugging appears on the screen element.

\subsection{Optical Dot Gain}

Deformation can be explained by what is visible with the light passing through the print material, where the light is reflected from the inner layers of the printing material. Colorimetric measurements are based on techniques where colours are measured as they are perceived by the human eye. Optical dot gain refers to the appearance of a given halftone, to the human eye or densitometer, when viewed at normal viewing conditions. This phenomenon is called "halo effect" and is perceived as an extension of the screen element. This illusion is generally caused by the paper and ink, or dot, interaction.

Fig. 3. Demonstration of optical dot gain

Densitometric techniques are basically a measurement of ink thickness that has been adapted to the inks used in print and where the processing of the measuring values are adapted to human perception, in relation to changes of lightness with 
varying ink film thicknesses. Such densitometers can determine not only the optical density, but also the parameters that characterize halftone printing, such as dot gain and relative printing contrast; the fact that ink acceptance/trapping can also be measured is an important feature in multi-colour printing. Both densitometric and colorimetric processes and instruments are used for colour measurements on the print. (Kipphan 2001)

\subsection{Print Control Strips}

Deformation of the screen elements can be easily detected by the print control strips. These test strips are a known means for the evaluation and control of print quality. Since a finished printed image generally consists of the overprint of the four halftone screens, it is very difficult to isolate each of the various components affecting the reproduction of the original image. To overcome this problem, a series of test elements can be printed along with the image and each element can be designed to highlight a particular aspect of the printing process. Some of these tests targets can be evaluated by eye, while others require the use of a densitometer. These test strips contain the various test elements for each colour, which can be evaluated on the basis of purely visual emotions or with colorimetric measurements. (Wyszecki \& Stiles, 1982)

The usual densitometric targets in a colour bar are: Solid Ink Density, Dot Area/Gain of the quarter, half and three-quarter tints, Contrast and the Trapping of ink overprints. Measuring strips serve for measuring and supplying the printing process in optimal working condition with the help of, the densitometer and spectrophotometer, and for keeping the circulation under optimal conditions. The divisions of such control strips used for the control of ink delivery are adjusted to the colour zone intervals of the printing press that we use. In each colour zone, there should be colour measurement fields. Only the measurable value allows an objective and to some extent, measure automated quality control in printing process. To establish and ensure constant print quality we measure the printed colour images to correct any deviation between the original proof prints and reproductions.

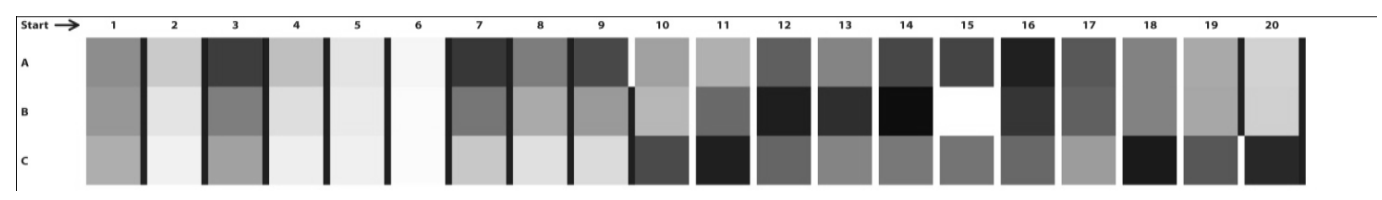

Fig. 4. Print control strips

\subsection{Parameters of Densitometry Valuation}

Valuation parameters are the measured factors that are found in the valuation process, for example, density and dot gain that determine the value of properties.

Density - absolute density is the measured density of the ink sample including the substrate, while relative density is the density of the ink sample minus the density of the substrate.

Dot Gain - monitors the way the dot is reproduced. As the image progresses through the reproduction process from Ctp to plate, plate to blanket and finally blanket to paper, the size of the dot changes. When we reproduce the tone withsystem 
Kumpar, D.; Zjakic, I. \& Bates, I.: Deviation of Deformation of the Screen Elemen...

of screening the surface under the print depends on the screen elements that have no theoretical coverage identical to the actual coverage. Increasing the actual coverage in relation to the theoretical coverage is called dot gain (Clapper \& Yule, 1953).

The first measurement of dot gain was provided in the 1930s by Murray, and followed with Murray-Davies equation

$$
F(V)=\frac{1-10^{-\left(D_{R}-D_{0}\right)}}{1-10^{-\left(D_{P P}-D_{0}\right)}} \times 100 \%
$$

Fig. 5. The Murray-Davies Dot Area Equation

$\mathrm{D}_{\mathrm{R}}-$ density of measured fields

$\mathrm{D}_{\mathrm{PP}}-$ density of full tone

$\mathrm{D}_{0}-$ whiteness of paper

Dot gain values obtains with subtracting the expected value $\mathrm{F}(\mathrm{A})$ of dot gain from the real values of dot gain $\mathrm{F}(\mathrm{V})$.

$$
\mathrm{DG} \%=\mathrm{F}(\mathrm{V})-\mathrm{F}(\mathrm{A})
$$

\section{Experiment}

This research was done on the print circulation of 35,000 copies. The measured elements of density of screeninig elements is in the fields of $25 \%, 50 \%, 75 \%$ and a field of the full tons that are needed to calculate the dot gain. Measurements were performed on an equal places for each copy. Test and measurements were made for each colour: Cyan, Magenta, Yellow and Black. For the calculation of dot gain curve, the measurements were carried out on of 5.000, 10.000, 15.000, 20.000, 25.000, 30.000 and 35.000 copy of prints.

Materials used in this study were the Norske Skog paper weight 42.5 gm-2 and printing plates from manufacturer of AGFA what is made on thermal CtP technology. To create a plate was used AM raster with line finesses of $42 \mu \mathrm{m}$. Print on the machine Manroland Geoman. Fountain solution is buffer Schwego Soft 5578 and artificially demineralized and artificially hardened with $\mathrm{H} 2 \mathrm{O} \mathrm{pH}=5.3, \mathrm{dh}=6.0$ with conductivity of $1640 \mathrm{mS}$. Measurement field is size of $3 \mathrm{~cm} \times 3 \mathrm{~cm}$ and measured are made with spectrophotometer "GretagMacbeth SpectroEye".

\section{Results and discussion}

After printing, the samples were measured and with this value we calculate the dot gain. The obtained measurement values are shown in tables and the results of dot gain are obtained with the graphs. The $\mathrm{x}$ axis shows the theoretical value of dot gain of $0-100 \%$, and the $\mathrm{Y}$ axis are shown the measured values of dot gain. 


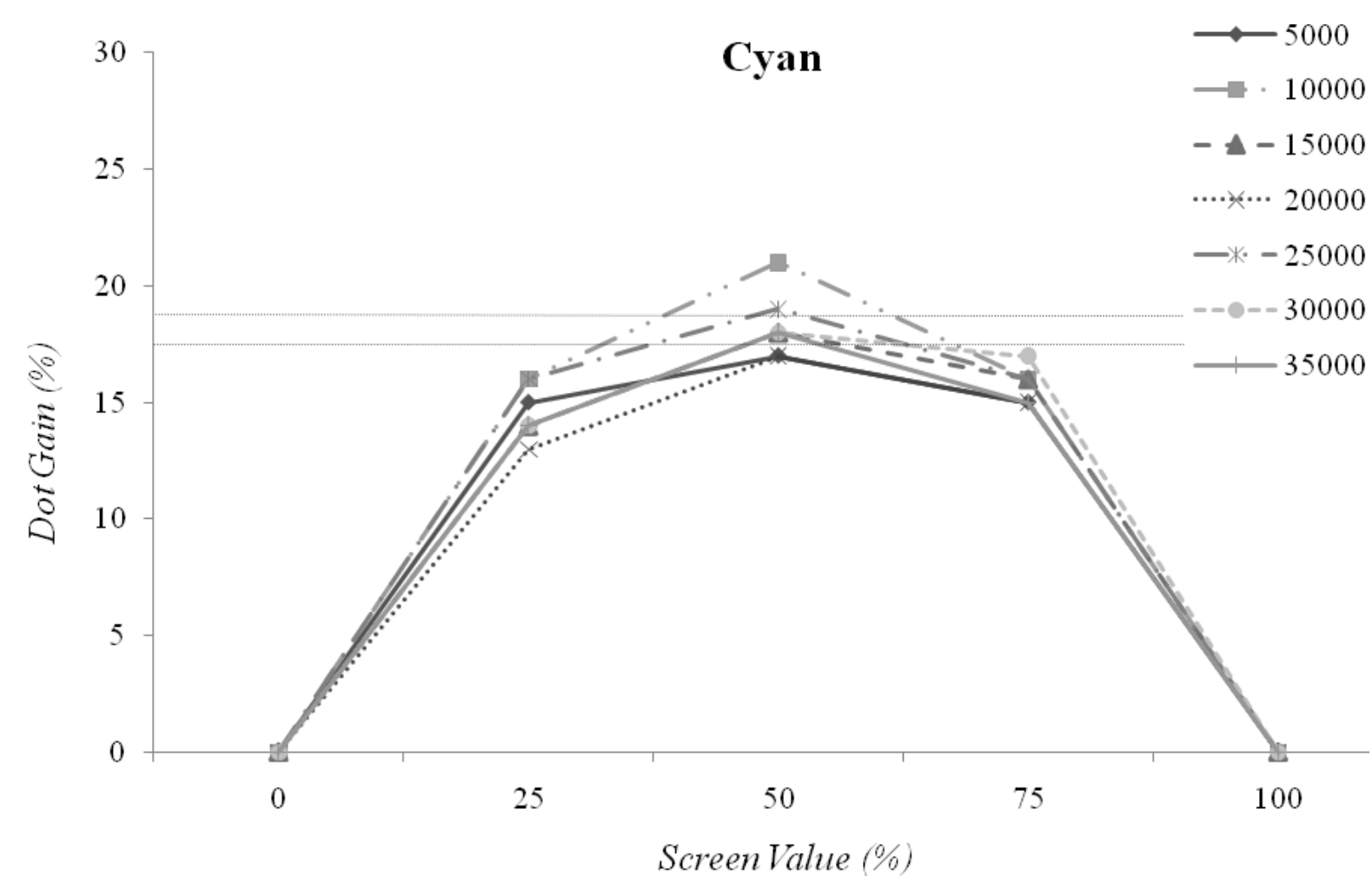

Fig. 6. Dot gain printed with Cyan colour during circulation of 35000 prints

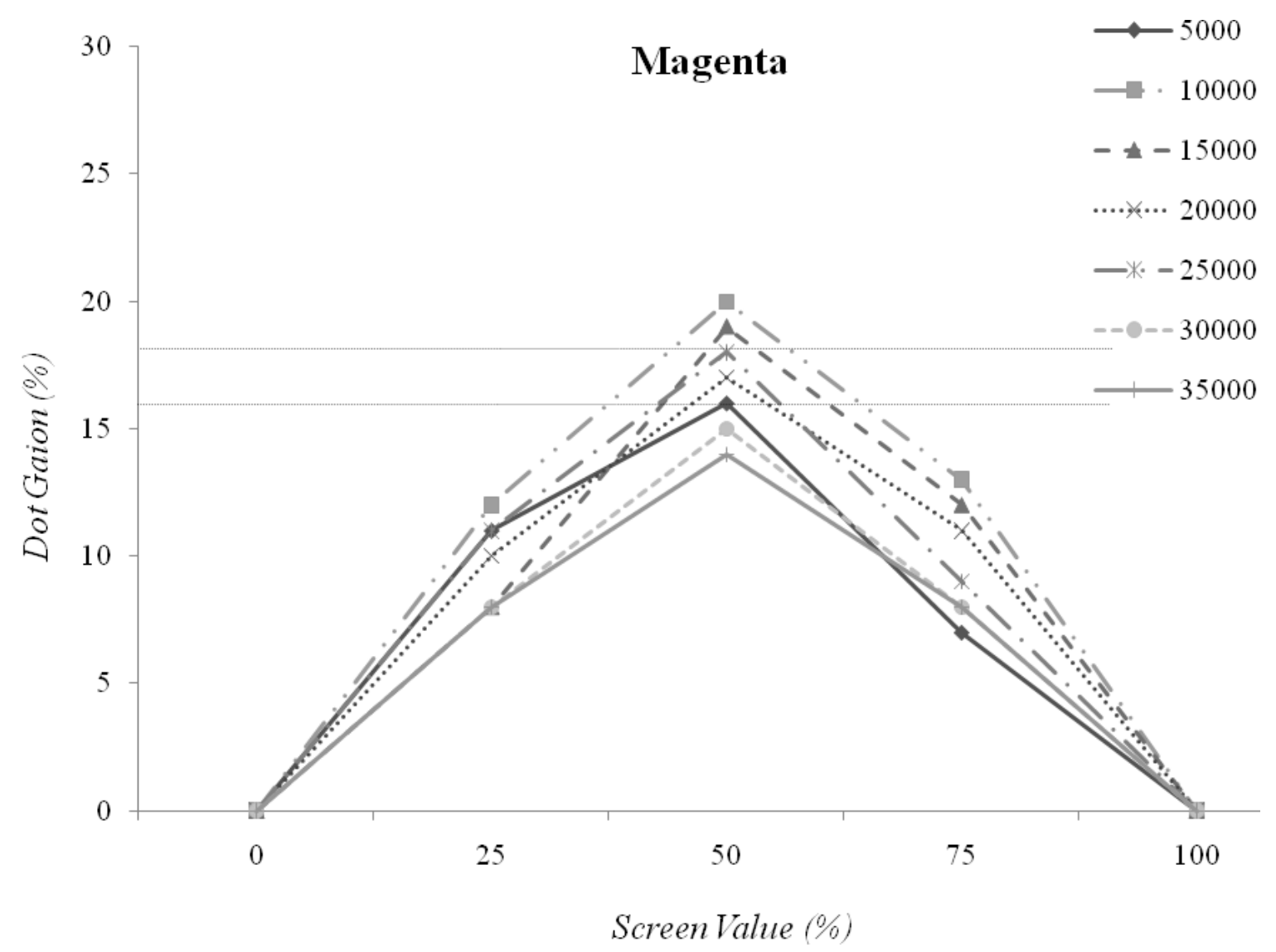

Fig. 7. Dot gain printed with Magenta colour during circulation of 35000 prints 
Kumpar, D.; Zjakic, I. \& Bates, I.: Deviation of Deformation of the Screen Elemen...

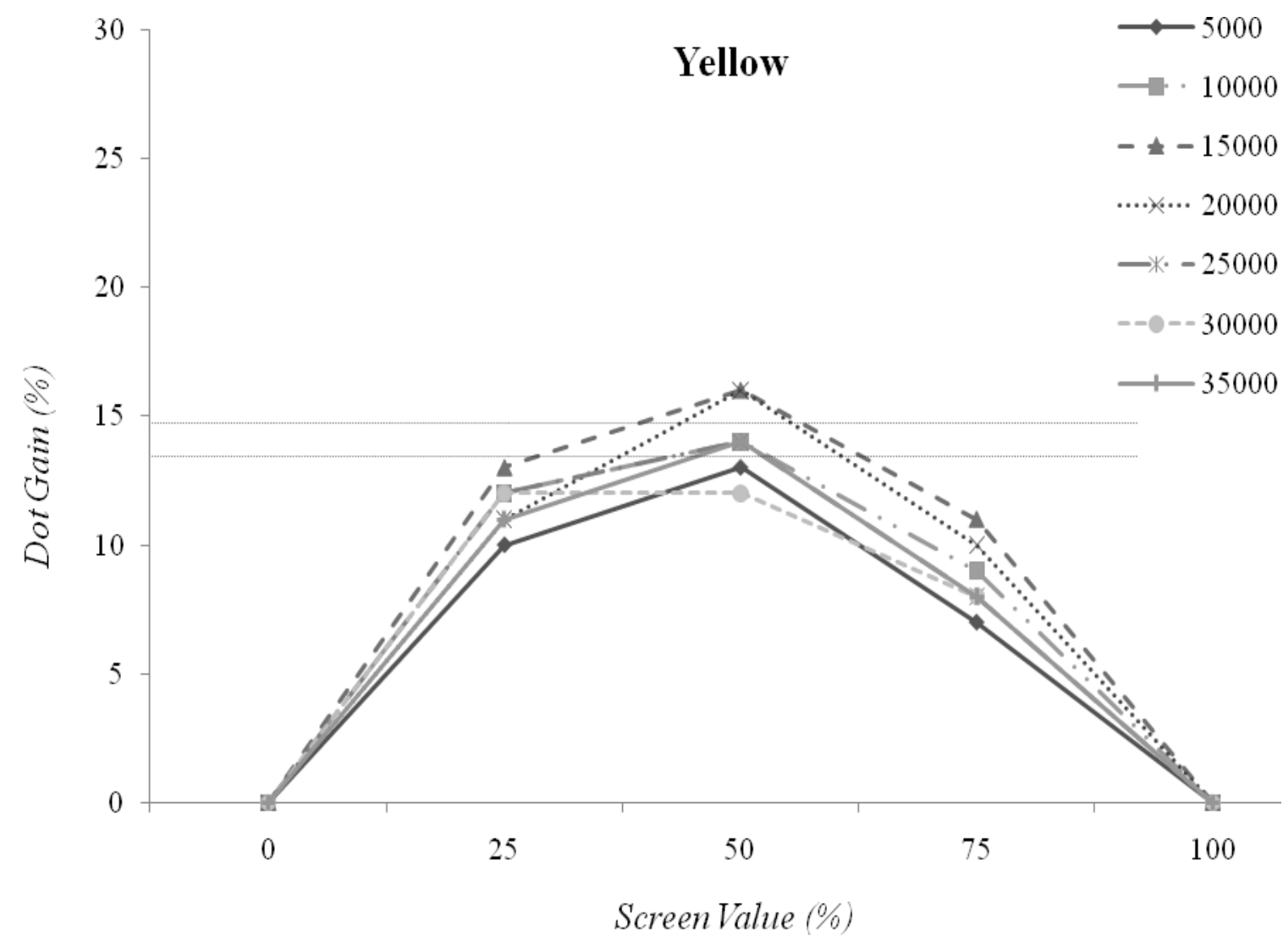

Fig. 8. Dot gain printed with Yellow colour during circulation of 35000 prints

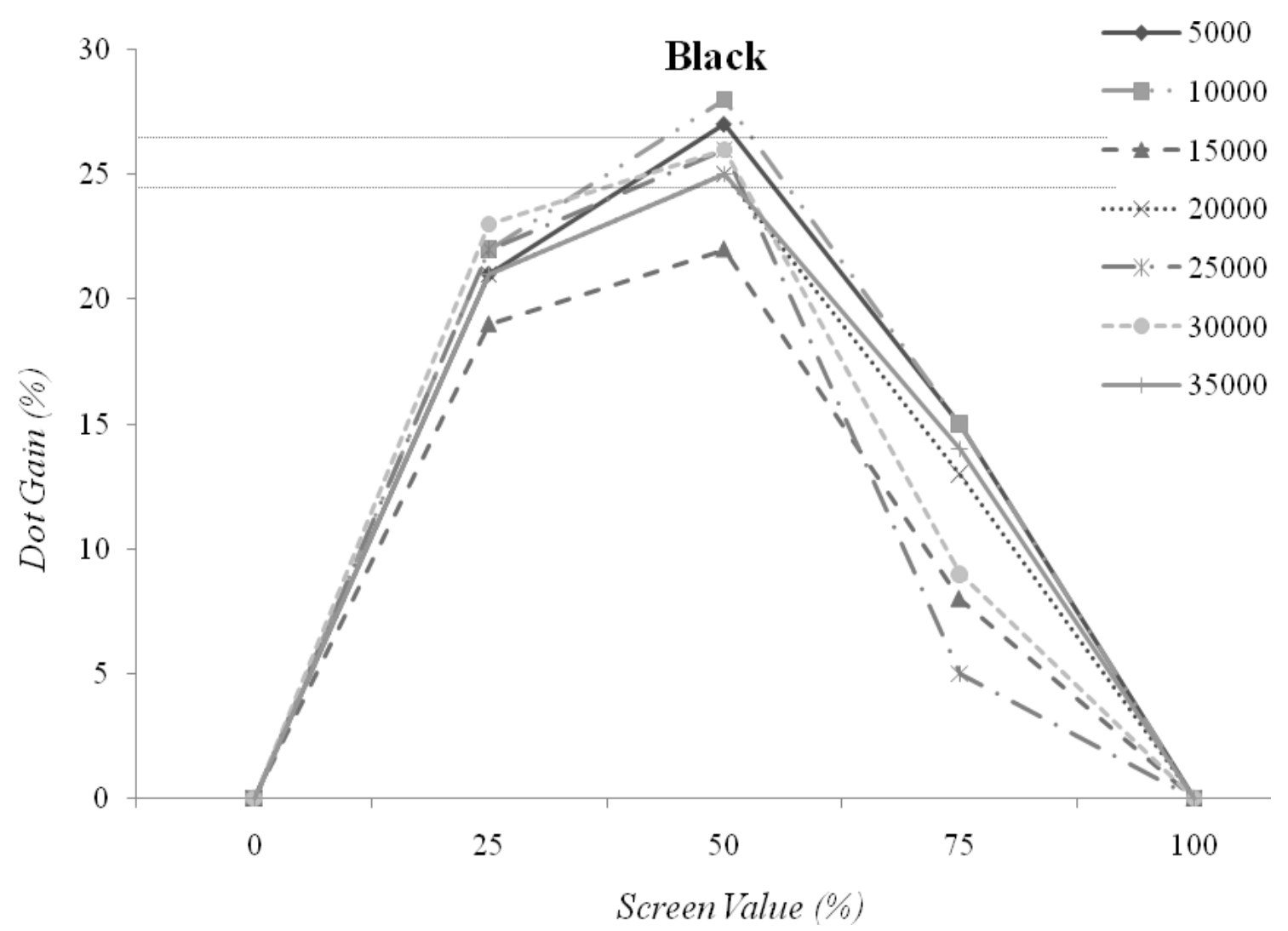

Fig. 9. Dot gain printed with Black colour during circulation of 35000 prints 


\begin{tabular}{|l|l|l|l|l|}
\hline & Cyan & Magenta & Yellow & Black \\
\hline standard deviation $=$ & 1,38013 & 2,16025 & 1,46385 & 1,90238 \\
\hline $\mathrm{x}=$ & 18,28571 & 17,00000 & 14,14286 & 25,57143 \\
\hline
\end{tabular}

Tab. 1. Standard deviation of prints

\begin{tabular}{|l|l|l|}
\hline \multirow{2}{*}{ Cyan (C) } & upper bound - & 18,9758 \\
\cline { 2 - 3 } & bottom bound - & 17,5956 \\
\hline \multirow{3}{*}{ Magenta (M) } & upper bound - & 18,0801 \\
\hline \multirow{3}{*}{ Yellow (Y) } & bottom bound - & 15,9199 \\
\hline \multirow{3}{*}{ Black (K) } & upper bound - & 14,8748 \\
\cline { 2 - 3 } & bottom bound - & 13,4109 \\
\hline
\end{tabular}

Tab.2. Indicator of deviation in upper and bottom boundaries

\section{Conclusion}

Dot gain is a phenomenon that in print production cannot be avoided, regardless of the printing technique we are working with. When it comes to newspaper production the dot gain is extremely important. Research in this paper proved that the value of dot gain oscillates during the print production through the circulation. It is also proven that the dot gain is different with each colour, which means that the dot gain depends on the rheological properties of colour and also on the other parameters, such as the printing press, pressure, drying paint, etc.

However, the tests found that the oscillations of dot gain varies from different colours. In fact, it is noted that the cyan colour dot gain diminishes as circulation increases except at the beginning of circulation when the dot gain is the smallest and increases suddenly, which subsequently leads to decreased dot gain that came to changing the colour temperature, viscosity of paint, etc. The question is why the dot gain was so great at the beginning of circulation. On the other hand, the size of the dot gain in the colour magenta as well changed, very much like the cyan colour, except that in this case the dot gain was greatest at the beginning of circulation.

Unlike the cyan and magenta colour, where there was a certain regularity in the changes in the size of dot gain, with the yellow and black such a principle does not exist. In these two colours it can be noticed that a change in dot gain is not dependent on circulation and does not behave the same as yellow and black colours.

To determine whether the dot gain oscillations is such, that there are no significant influences on the quality, we calculated the standard deviation of the dot 
Kumpar, D.; Zjakic, I. \& Bates, I.: Deviation of Deformation of the Screen Elemen...

gain in circulation of 35.000 pieces. It was found that the standard deviation is much smaller than the oscillations of dot gain, which provided that the standard deviation was performed for raster value of $50 \%$ as recommended by international guidelines.

The standard deviation of the dot gain for all colour is not the same in different colours. The above can be explained that the conditions in the press at those printing machine are different in different printing unit. The fact that there are large oscillations of dot gain during printing process through circulation proves that during printing it is necessary to pay greater attention to the print circulation, but also shows that it is necessary to calibrate and characterize the entire printing system to the value of dot gain uniform and also that oscillations of dot gain through the circulation was smaller. Setting up the production system as stated earlier, is helping to standardize the production, but such standardization does not guarantee that in newspaper production it will make a quality product.

The reason for this is that there are virtually no laws that determine exactly when the dot gain will be greater or when less. However, there are observations, such as in our work, and proven information that some colours may have a similar change in the value of dot gain, such as cyan and magenta in this study. These findings lead to new tasks that need to be scientifically established concerning the above parameters. The conclusions we found, lead us to the fact that it is necessary to make further investigation of other parameters and to try to determine whether permitted tolerances are really enough to prevent change in dot gain, which reduces the quality of newspaper printing.

The following tests will attempt to synthesize the results of previous tests and the results of the diversity parameters of the machine on which it prints the newspaper. New concepts will be able to conclude the parameters and the extent to rebrand dot gain during printing, and thus will enable the production of quality newspaper products.

\section{References}

Clapper, F. R. \& Yule, J. A. C. (1953). The effect of multiple internal reflections on the densities of half-tone prints on paper, Journal of the Optical Society of America, 43(7), 600

Kipphan, H. (2001). Handbook of Print Media, Springer, ISBN 3-540-67326-1, Heidelberg

Preston, J. S. \& Gate, L. F.The influence of colour and surface topography on the measurement of effective refractive index of offset printed coated papers, Colloids and Surfaces A: Physicochemical and Engineering Aspects, 252, 20, January, 2005, 99-104

Verikas, A. \& Bacauskiene, M. (2008). Estimating ink density from colour camera RGB values by the local kernel ridge regression, Engineering Applications of Artificial Intelligence, 21, Issue 1, February 35-42

Wyszecki, G. \& Stiles, W. S. (1982). Color Science: Concepts and Methods, Quantitative Data and Formulae, second ed., Wiley-Interscience Publication, ISBN: 0-471-02106-7, New York, 1982 